\title{
Acquired hemophilia A: emerging treatment options
}

\author{
This article was published in the following Dove Press journal: \\ Journal of Blood Medicine \\ 8 May 2015 \\ Number of times this article has been viewed
}

\author{
Maissaa Janbain' \\ Cindy A Leissinger' \\ Rebecca Kruse-Jarres ${ }^{2}$ \\ 'Louisiana Center for Bleeding and \\ Clotting Disorders, Tulane University \\ Medical Center, New Orleans, \\ LA, USA; ${ }^{2}$ Washington Center for \\ Bleeding Disorders, Blood Works \\ NW, Seattle, WA, USA
}

\begin{abstract}
Acquired hemophilia $\mathrm{A}$ is a rare autoimmune disorder caused by an autoantibody (inhibitor) to factor VIII (FVIII) that interferes with its coagulant function and predisposes to severe, potentially life-threatening hemorrhage. Disease management focuses on controlling bleeding, primarily with the use of bypassing therapy and recombinant porcine FVIII, and permanently eradicating the autoantibody using various immunosuppressants. Treatment challenges include delayed diagnosis, difficulty achieving hemostasis and durable remissions, and complications associated with the use of hemostatic and immunosuppressive therapy in a primarily older patient population.
\end{abstract}

Keywords: autoantibodies, factor VIII, hemostasis, inhibitors, inhibitor eradication

\section{Introduction}

Acquired hemophilia A (AHA) is a rare autoimmune disease caused by immunoglobulin $\mathrm{G}$ antibodies that bind to specific domains on the factor (F) VIII molecule, partially or completely neutralizing its coagulant function. ${ }^{1-3}$ Because FVIII serves as a cofactor to activated FIX in the tenase complex, its deficiency reduces thrombin generation on the surface of activated platelets, ${ }^{4}$ predisposing to bleeding that may be life-threatening. ${ }^{1,2}$ The incidence of AHA increases with age, ranging from an estimated 0.045 per million per year in children $<16$ years to 14.7 per million annually in people $>85$ years. ${ }^{5}$ Approximately $10 \%$ of persons with AHA are younger women diagnosed during or after pregnancy, while more than $80 \%$ of those affected are men and women 65 years and older (median age: 78 years).$^{5-9}$ Roughly, half of all AHA cases are attributable to an underlying medical condition, typically another autoimmune disorder, malignancy, or a drug/allergic reaction (Table 1); the remainder are idiopathic..$^{5-8}$

The usual clinical presentation of AHA is spontaneous or provoked bleeding and an unexplained, prolonged activated partial thromboplastin time (aPTT) in a person with a negative personal or family history of a coagulopathy. ${ }^{10,11}$ In contrast to the joint bleeds that characterize congenital hemophilia A, ${ }^{12}$ bleeding associated with AHA usually manifests as spontaneous subcutaneous hematomas and extensive bruising (Figure 1), although muscle bleeding, hematuria, epistaxis, gastrointestinal bleeding, and even intracranial hemorrhage may occur. ${ }^{8,10,11}$ A substantial number of individuals - nearly $7 \%$ of the 501 patients enrolled in the European Acquired Haemophilia Registry (EACH2), the largest reported AHA observational dataset ${ }^{8}-$ had no bleeding symptoms, and the diathesis was detected incidentally after routine blood testing. ${ }^{10,11}$
Correspondence: Rebecca Kruse-Jarres Washington Center for Bleeding Disorders, Blood Works NW,

921 Terry Ave, Seattle, WA 98104, USA

Tel +l 2066896570

Email rebeccakr@psbc.org 
Table I Underlying conditions associated with AHA

\section{Pregnancy}

Autoimmune diseases

- Systemic lupus erythematosus

- Rheumatoid arthritis

- Multiple sclerosis

- Dermatologic conditions (eg, psoriasis, pemphigus vulgaris)

- Temporal arteritis

- Sjögren syndrome

- Inflammatory bowel disease

- Goodpasture syndrome

- Graft-versus-host disease

- Myasthenia gravis

- Graves' disease

- Autoimmune hemolytic anemia

- Autoimmune hypothyroidism

Underlying malignancy

- Hematologic malignancy (eg, chronic lymphocytic leukemia, non-Hodgkin lymphoma, multiple myeloma, Waldenström macroglobulinemia)

- Solid organ tumors (eg, lung, prostate, pancreas, breast)

Medications

- Antibiotics (eg, penicillin, sulfonamides)

- Others (eg, phenytoin, chloramphenicol, methyldopa, interferon alpha, fludarabine)

Acute hepatitis B and C infections

Respiratory disease (eg, asthma, chronic obstructive pulmonary disease)

Note: Data from Toschi and Baudo ${ }^{3}$ and Sborov and Rodgers. ${ }^{18}$

Abbreviation: AHA, acquired hemophilia A.

The management of AHA requires a two-pronged, parallel approach: 1) control bleeding and 2) eradicate the inhibitor. ${ }^{1,713,14}$ Additionally, an underlying cause should be investigated. Here we review the strategies used to treat patients with AHA and discuss newer treatments and emerging therapies that may surmount some of the challenges posed by this complex bleeding disorder.

\section{Hemostatic therapy}

The need for hemostatic therapy in patients with AHA is determined by bleeding site and severity. ${ }^{13-16}$ The inhibitor

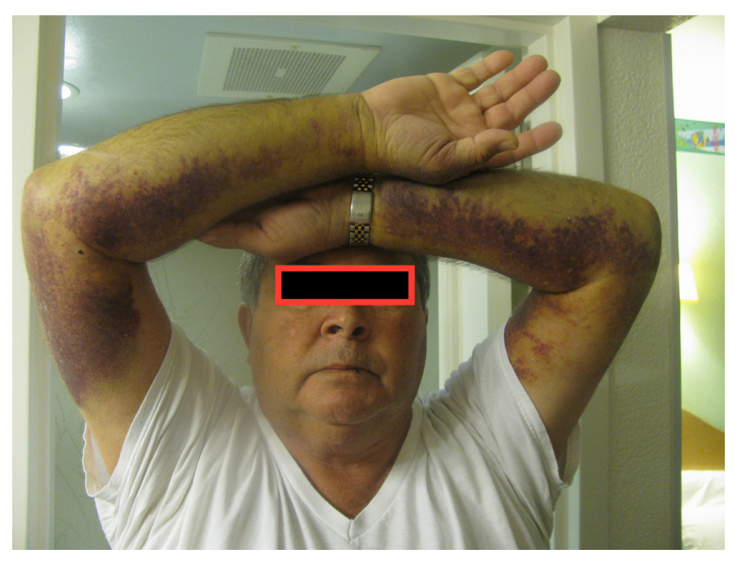

Figure I Bleeding associated with acquired hemophilia A. Note: Extensive ecchymosis of the arms is visible. titer provides little guidance for clinical decision making, as it does not correlate with bleeding phenotype. Unlike the alloantibodies associated with congenital hemophilia A, which inactivate FVIII in direct proportion to their concentration (type 1 kinetics), acquired inhibitors exhibit type $2 /$ nonlinear kinetics, whereby rapid initial inactivation is followed by a slower phase of equilibrium., ${ }^{4,11,17}$ Some measurable FVIII (usually $<10 \%$ of normal) is often detected in patients with high-titer anti-FVIII antibodies ( $>5$ Bethesda units [BU]), but this small amount of residual circulating FVIII offers no protection from bleeding. In fact, residual FVIII activity levels as high as $10 \%$ - consistent with a diagnosis of mild congenital hemophilia A in which bleeding seldom occurs - have been observed in the presence of severe hemorrhage. ${ }^{11}$

\section{Minor bleeding}

In persons with low-titer acquired inhibitors ( $\leq 5 \mathrm{BU})$ who have no/minimal bleeding and do not require surgery, observation and laboratory monitoring may be sufficient. ${ }^{18}$ Even extensive subcutaneous hemorrhage can usually be managed without treatment, although patients may be hospitalized to facilitate close monitoring. ${ }^{19}$ Inhibitors spontaneously disappear within a few months in approximately $25 \%$ of cases, primarily those associated with pregnancy or antibiotic therapy. ${ }^{2,20}$

When minor bleeding requires treatment, initial interventions include routine hemostatic techniques; avoidance of invasive procedures; and discontinuation of any therapies that can exacerbate bleeding (ie, antiplatelet or anticoagulant drugs), if at all possible. For patients with an FVIII level $>5 \%$ and a very low-titer inhibitor $(<2 \mathrm{BU})$, desmopressin (DDAVP) may cause a transient rise in FVIII levels capable of controlling minor bleeding. ${ }^{1,4,14}$ Human FVIII is more likely than DDAVP to achieve hemostasis in patients with low-titer inhibitors, but large, even massive doses may be required to saturate the autoantibody. Human FVIII is almost never effective in patients with high-titer inhibitors.

\section{Major bleeding}

Severe bleeding symptoms on presentation have been reported in up to $72 \%$ of patients and require the prompt initiation of antihemorrhagic treatment. ${ }^{21}$ Human FVIII concentrates or DDAVP may have some value as first-line treatment if the inhibitor titer is $\leq 5 \mathrm{BU}$ but will only control bleeding to the degree that hemostatic circulating levels of FVIII can be achieved. Thus, these interventions are likely 
to be completely ineffective in patients with high-titer autoantibodies.

\section{Bypassing agents}

Bypassing therapy with agents that circumvent the need for FVIII is the current treatment of choice for major bleeding in patients with high-titer ( $>5 \mathrm{BU})$ acquired inhibitors. ${ }^{13,15}$ Two bypassing agents are available: plasma-derived activated prothrombin complex concentrate (aPCC) (FEIBA; Baxter Healthcare Corporation, Deerfield, IL, USA), which contains exogenously activated FII, FVII, FIX, and FX, and recombinant activated FVII (rFVIIa) (Novo Seven; Novo Nordisk A/S, Bagsværd, Denmark). Both achieve hemostasis by generating thrombin (in the absence of FVIII) at the site of bleeding, ${ }^{22,23}$ but they differ with respect to their biochemical properties and pharmacokinetics. In particular, aPCC has a longer half-life of 4-7 hours ${ }^{24}$ compared with approximately 2 hours for rFVIIa. ${ }^{25}$

Although good and equivalent success rates have been reported for aPCC and rFVIIa used for hemostatic management in $\mathrm{AHA},{ }^{26}$ neither is predictably effective. In the EACH2, bleeding was controlled in 60 of 64 patients (93\%) treated with aPCC first-line and 159 of 174 (91\%) of those treated with rFVIIa first-line. ${ }^{26}$ Additionally, patients may exhibit a differential response to treatment. A randomized crossover study comparing aPCC and rFVIIa in the management of joint bleeding associated with congenital hemophilia $\mathrm{A}$ and inhibitors found that some patients reported better bleed resolution with one bypassing agent than the other, ${ }^{27}$ and in our clinical practices, we have also observed this phenomenon in patients with AHA.

Either bypassing agent can be used first-line for bleeding in AHA, with choice determined by a patient's previous response (if any), dosing convenience, possible need for early redosing, preference for a plasma-derived or recombinant product, and availability. ${ }^{14}$ Mucosal bleeds may benefit from the addition of an antifibrinolytic drug (ie, epsilonaminocaproic acid or tranexamic acid), with accumulating data supporting the safety of concomitant treatment, even with aPCC. ${ }^{28}$ If first-line therapy fails, treatment with the alternative bypassing agent should be quickly initiated and may prove successful. The use of higher than recommended doses of aPCC (100 U/kg per dose or $200 \mathrm{U} / \mathrm{kg} /$ day) or rFVIIa (90 $\mu \mathrm{g} / \mathrm{kg}$ every 2 hours) should be considered only under exceptional circumstances because of the increased risk for arterial and venous thrombosis. ${ }^{14,29}$ Such risk may be heightened in patients with AHA, who are frequently older and have comorbidities, such as cardiovascular disease, cancer, and pregnancy, that increase thrombotic risk. ${ }^{13,30,31}$ The combined use of both bypassing agents should be avoided except in the setting of life-threatening bleeding uncontrolled by either agent used alone. ${ }^{32}$

No laboratory tests have been validated for monitoring the efficacy of bypassing therapy. Treatment is assessed clinically, which is often difficult due to the location of bleeding. Although global hemostatic assays have been evaluated as a strategy for measuring thrombin generation after the application of bypassing therapy, these measurements do not correlate with clinical efficacy or bleed resolution. ${ }^{33}$

Once hemostasis has been achieved, ongoing treatment at reduced dose (as prophylaxis) may be needed to prevent recurrence of bleeding.

\section{Case study I: challenges to achieving hemostasis - part I}

A 74-year-old woman with hypertension, type 2 diabetes mellitus, hyperlipidemia, and chronic obstructive pulmonary disease presents to the emergency room with right leg swelling. Deep vein thrombosis is suspected, but a venous Doppler ultrasound is negative, and she is released home. She returns 2 days later with worsening leg swelling, tingling in her foot, cold and bluish toes, and absent pedal pulses. Ultrasound shows a large intramuscular hematoma; compartment syndrome is diagnosed, and she is taken to the operating room for emergent release. Significant intraoperative bleeding occurs, and active bleeding from the fasciotomy incision continues in the recovery room. Laboratory studies show a hemoglobin of $5.6 \mathrm{~g} / \mathrm{dL}$, a prolonged aPTT of 72 seconds, a FVIII level of $<1 \%$, and an inhibitor titer of 134 BU. She is diagnosed with AHA.

The patient is given 4 units of packed red blood cells (RBCs), and hemostatic therapy is started with rFVIIa $90 \mu \mathrm{g} / \mathrm{kg}$ every 2 hours. After 6 hours, the patient is still actively bleeding, and the hemostatic regimen is changed to aPCC $75 \mathrm{U} / \mathrm{kg}$ every 6 hours. Active bleeding persists 24 hours after switching products, and another RBC transfusion is given. Combined sequential bypassing therapy is initiated using alternating doses of aPCC and rFVIIa (rFVIIa dosing 6 hours after aPCC and then aPCC dosing 2 hours after rFVIIa) (Figure 3).

Bleeding continues unabated after 24 hours of combined sequential therapy. Another RBC transfusion is given and intravenous tranexamic acid $10 \mathrm{mg} / \mathrm{kg}$ every 6 hours is added. Bleeding persists despite maximum doses of hemostatic agents, and there is growing concern about the potential for thrombotic complications, given the patient's age and comorbidities. 


\section{Porcine FVIII}

Despite the prompt initiation of appropriate bypassing therapy, fatal hemorrhage occurs in 3\%-8\% of patients with AHA., ${ }^{5,8}$ In the past, a highly purified plasma-derived porcine FVIII (Hyate:C; Speywood Pharmaceuticals Ltd, Maidenhead, Berks, UK) was an option for the management of bleeding caused by acquired inhibitors. In clinical practice, the hemostatic efficacy of porcine FVIII was $90 \%$, and inhibitory antibodies against hFVIII showed low cross-reactivity with porcine FVIII $(\sim 15 \%){ }^{34,35} \mathrm{~A}$ major advantage to the use of porcine FVIII compared with bypassing agents was the ability to measure circulating FVIII levels using a standard FVIII assay, thus allowing dose adjustment. Manufacture of this product ceased in 2004 because of concerns about possible residual viral contamination, primarily by porcine parvovirus, ${ }^{36}$ leaving a void in the AHA treatment armamentarium.

A recombinant porcine sequence FVIII (rpFVIII) (Obizur; Baxter) formulated without animal-derived products has recently received US Food and Drug Administration approval for use in AHA. The pivotal Phase II/III clinical trial that led to product licensure enrolled 28 adults with AHA (age range: $40-\geq 65$ years) experiencing serious bleeding episodes that required hospitalization. ${ }^{37}$ Following administration of an initial rpFVIII dose of $200 \mathrm{U} / \mathrm{kg}$, therapeutic FVIII activity levels were achieved and maintained with intermittent rpFVIII administration until bleeding resolved. Response was determined by clinical assessment and FVIII activity levels. A positive response at 24 hours, defined as effective or partially effective bleeding control as determined by clinical assessment and FVIII activity levels, was reported for all 28 patients, and treatment of the initial bleeding episode was successful in 24 of 28 (86\%). (The remaining four patients were taken off study before a final assessment could be made.) No thrombotic or other serious adverse events occurred that were related to the study drug. At baseline and before exposure to rpFVIII, ten of 28 patients ( $36 \%$ ) demonstrated anti-rpFVIII antibody activity (0.8-29 BU) due to cross-reactivity with the patients' existing FVIII autoantibodies. Antibody status was not known before initiation of treatment with rpFVIII, however, and all study patients achieved FVIII levels $>100 \%$, although higher doses were required in those with anti-rpFVIII antibodies at baseline. Four patients without baseline antibodies eventually developed anti-porcine inhibitors (range: 8-166 BU), and treatment was subsequently discontinued in two of these individuals.

\section{Case study I: challenges to achieving hemostasis - part 2}

The patient is given rpFVIII at $200 \mathrm{U} / \mathrm{kg}$. Her FVIII level rises from $<1 \%$ to $76 \% 1$ hour after infusion, and she receives additional doses every 2-3 hours for the first 24 hours to maintain FVIII levels $>80 \%$. Overall, she has good clinical bleeding control at 24 hours. Bolus doses of rpFVIII are continued every 4-6 hours, with a target trough level of $>70 \%$. The fasciotomy incision begins to heal by secondary intention, and bleeding continues to be well controlled. rpFVIII dosing continues, with the goal of maintaining FVIII levels $>25 \%$ to ensure wound healing and prevent rebleeding.

After 100 rpFVIII doses, FVIII recovery is less than expected, and an anti-rpFVIII antibody measuring $20 \mathrm{BU}$ is detected. At that point, bleeding is controlled, and the patient is transitioned to aPCC (75 IU/kg/day). The wound continues to heal by primary intention with no further bleeding.

\section{Emerging hemostatic therapies}

A recombinant humanized anti-FIXa/FX bispecific antibody has been studied in a primate model of severe AHA..$^{38}$ The investigational agent brings FIX and FX into spatially appropriate position, mimicking the cofactor function of FVIII without the need for activation. Weekly subcutaneous doses of the experimental agent (ACE910) significantly prevented spontaneous bleeding in all four treated cynomolgus monkeys (one animal experienced traumatic bleeding), and no hematuria or organ bleeding was seen at necropsy. Shortening of the prolonged aPTT observed immediately after the initial injection gradually disappeared in three of four monkeys in the middle of the dosing period, suggesting the development of alloantibodies to ACE910. In another primate model of acute bleeding, the hemostatic efficacy of a single $1-3 \mathrm{mg} / \mathrm{kg}$ bolus dose of ACE910 was equivalent to twice-daily doses of rpFVIII 10 U/kg. Because ACE910 has a 3-week half-life and nearly $100 \%$ subcutaneous bioavailability, once-weekly dosing to maintain hemostatic trough levels is feasible. Clinical investigation of ACE910 is ongoing.

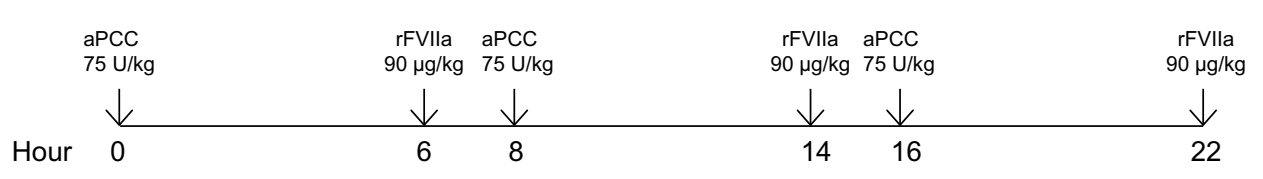

Figure 2 Regimen for combined sequential bypassing therapy. 
Augmenting the activity of activated FV (FVa) is another novel approach for bypassing inhibitors in congenital hemophilia A. As a cofactor in the prothrombinase complex, FVa enhances the rate of thrombin generation by approximately 10,000-fold; however, this clotting factor is rapidly inactivated by protein $\mathrm{C} .{ }^{39,40}$ super $\mathrm{FVa}$ is a molecularly engineered FVa variant in which an interdomain disulfide bond covalently anchors the A2 to the A3 domain, thus providing increased cofactor activity, while protein $\mathrm{C}$ cleavage site mutations enhance biologic stability. ${ }^{41,42}$ When evaluated in in vitro studies in hemophilia plasma with and without inhibitors and in in vivo mouse models, SuperFVa had excellent hemostatic properties. ${ }^{41}$ All prohemostatic effects, including clot stability and prolongation of clot lysis, were synergistically enhanced when SuperFVa was used in combination with trace amounts of FVIIa in hemophilic or normal plasma with inhibitors. ${ }^{43}$

\section{Inhibitor eradication}

Immunosuppression to eradicate an acquired inhibitor should begin immediately upon diagnosis in all patients. ${ }^{13}$ Even those who are not actively bleeding remain at risk for life-threatening hemorrhage until the autoantibody has been eliminated, ${ }^{5}$ and overall survival is improved in patients who achieve a complete response to inhibitor eradication. ${ }^{7}$

\section{Steroids and cyclophosphamide}

Although the optimal immunosuppressive regimen and duration of treatment remains to be determined, prednisone (1 $\mathrm{mg} / \mathrm{kg} /$ day) alone or in combination with oral cyclophosphamide (50-100 mg/day) is currently recommended as first-line treatment for autoantibody eradication. ${ }^{13-15}$ (Azathioprine, 6-mercaptopurine, and vincristine may be considered as alternatives to cyclophosphamide for cytotoxic therapy)., ${ }^{2,44} 46$ In the EACH2 that included data on first-line immunosuppression for 276 patients, those given steroids were compared with patients receiving steroids plus cyclophosphamide. ${ }^{47}$ The groups were matched by logistic regression and a propensity score for age, sex, inhibitor titer, FVIII level, and underlying etiology. Among patients treated with steroids alone, 48\% (68 of 142) achieved a stable complete remission (CR), as compared with $70 \%$ (58 of 83) given combination therapy (median time to $\mathrm{CR}$ for both regimens: $\sim 5$ weeks). These findings are in agreement with several other reports showing that first-line treatment with steroids plus cyclophosphamide is more likely to achieve a CR than steroids alone. , $^{5,78,49}$ Nonetheless, the final outcome of treatment, as measured by survival and sustained remission, was the same for both regimens in all large studies. ${ }^{5,7,47}$
Successful eradication is defined as an undetectable inhibitor $(<0.6 \mathrm{BU})$ and normal FVIII levels $(>50 \%) .{ }^{15}$ If the FVIII level is not increasing and the inhibitor titer is not dropping after 3-5 weeks of treatment, second-line immunosuppression should begin. ${ }^{14}$

\section{Case study 2: challenges to inhibitor eradication - part I}

A 62-year-old woman presents to the emergency room with multiple large bruises on her lower and upper extremities and a 2-day history of severe left hip pain that radiates into her back and lower abdomen, worsens with walking and leg extension, and improves with hip and knee flexion. Her medical history includes a diagnosis of systemic lupus erythematosus that was intermittently treated with corticosteroids (the last time approximately 2 years ago). She has experienced periodic rashes over most of her body for the last 5 years and reports increased bruising in the past 2 months. Computed tomography reveals a large retroperitoneal hematoma and bleeding into the iliopsoas plane. The potential for femoral nerve compression from an expanding iliopsoas bleed is of major concern. Hemoglobin is $5.5 \mathrm{~g} / \mathrm{dL}$, and she is given 6 units of RBCs. The prothrombin time is normal, but the aPTT is markedly elevated at 89 seconds and is not corrected by incubation of patient's plasma with normal plasma at $37^{\circ} \mathrm{C}$ for 2 hours (mixing study). The diagnosis of AHA is confirmed by an FVIII level $<1 \%$ and a Bethesda assay showing an inhibitor titer of 1,200 BU.

Hemostatic therapy is initiated with rFVIIa $90 \mu \mathrm{g} / \mathrm{kg}$ every 3 hours, and bleeding symptoms stabilize within 24 hours. Over the next 2 days, dosing frequency is gradually reduced to every 8 hours, but bleeding recurs when the dosing interval is further extended. Daily immunosuppressive therapy with oral prednisone $1 \mathrm{mg} / \mathrm{kg}$ and cyclophosphamide $1 \mathrm{mg} / \mathrm{kg}$ is started immediately upon diagnosis. During the first 5 days of treatment, the inhibitor titer decreases to $829 \mathrm{BU}$ and holds at this level for the next 2 weeks.

The patient remains hospitalized because of the need for ongoing hemostatic therapy.

\section{Rituximab}

The anti-CD20 monoclonal antibody rituximab is increasingly used first- and second-line for inhibitor eradication in AHA. No data support the contention that rituximab used alone or in combination with other immunosuppressants results in higher remission rates or more rapid remissions. ${ }^{14}$ In the EACH2, a stable CR was achieved by $59 \%$ of patients ( 30 of 51 ) treated with any rituximab - a 
success rate halfway between that achieved with steroids alone (48\%) and steroids plus cyclophosphamide (70\%). ${ }^{47}$ Nevertheless, some patients resistant to standard first-line immunosuppression respond to second-line rituximab. In the EACH2, seven of 14 patients (50\%) achieved a stable $\mathrm{CR}$ with rituximab-based regimens used after the failure of first-line $\operatorname{drug}(\mathrm{s})$ or relapse.

\section{Other immunosuppressants}

Calcineurin inhibitors (eg, cyclosporine, tacrolimus) and mycophenolate mofetil are alternatives to rituximab in patients who do not respond to first-line treatment. ${ }^{14}$ The addition of immune tolerance with FVIII may be considered, although the benefits are unclear, and such treatment significantly increases the cost of inhibitor eradication. Available evidence does not support the use of intravenous immunoglobulin for the elimination of acquired inhibitors. ${ }^{5,747}$

On the basis of data from EACH2, approximately $50 \%$ of patients can be expected to achieve a stable remission with second-line therapy using a variety of immunosuppressive agents. $^{47}$

\section{Case study 2: challenges to inhibitor eradication - part 2}

The patient receives rituximab $375 \mathrm{mg} / \mathrm{m}^{2}$ weekly for 4 weeks, and prednisone and cyclophosphamide are slowly tapered. Her inhibitor titer ranges between 700 to $800 \mathrm{BU}$ for the next 4 weeks, after which it slowly decreases and becomes undetectable over another 4-week period. At 1-year follow-up, her B-cells are reconstituted, FVIII remains in the normal range, and the autoantibody is undetectable.

\section{Follow-up}

Long-term laboratory follow-up is needed to demonstrate a stable CR and rule out relapse, which occurs in up to $20 \%$ of patients. ${ }^{13,14,19}$ Monthly monitoring of aPTT and FVIII activity (FVIII:C) for the first 6 months, then every 2 to 3 months up to 12 months, and every 6 months during the second year and possibly beyond - is recommended. ${ }^{15}$ Patients should be educated about the signs and symptoms of recurrence and urged to promptly report any bleeding or bruising.

Remission of AHA is often associated with high FVIII levels. Because many patients have thromboembolic risk factors associated with comorbidities, discontinuation of antiplatelet or antithrombotic therapy during bleeding, and/or reduced mobilization due to hospitalization, pharmacologic thromboprophylaxis is recommended after a stable CR is achieved. ${ }^{15}$

\section{Summary, future directions, and perspective commentary}

The rarity of AHA means that patients usually present to clinicians with no previous experience in its management, often resulting in delays in diagnosis and treatment. Owing to the high morbidity and mortality associated with the condition and the complexities of management, immediate consultation with a hemophilia treatment center experienced in the care of inhibitor patients is recommended whenever AHA is suspected, whether or not bleeding is present.

Achieving hemostasis and eliminating the inhibitor represent the core of AHA management. The return of porcine FVIII has been eagerly awaited by clinicians and is now available as a recombinant concentrate. Promising new investigational agents may soon provide additional options for successfully controlling serious bleeding episodes in patients with FVIII autoantibodies. Although inhibitor eradication is successful in most individuals with AHA, immunosuppression in this primarily older patient population is associated with significant side effects, including sepsis, neutropenia, diabetes, and psychiatric illness. ${ }^{47}$ Development of less toxic and more targeted immunosuppressive regimens capable of achieving faster, more durable responses remains a critical unmet need in AHA therapy.

\section{Acknowledgment}

Michele Grygotis, an independent consultant, provided medical writing services that were funded by Tulane University School of Medicine.

\section{Disclosure}

RKJ has received research support from Baxter Bioscience and Biogen Idec and received honoraria for advisory board participation and consulting fees from Baxter Bioscience, Bayer Healthcare, Grifols, and Novo Nordisk. MJ received honoraria for advisory board participation from Bayer and CSL Behring. CAL has received research funding from Baxter, Bayer, CSL Behring, and Novo Nordisk and received honoraria for advisory board participation from Baxter, Bayer, Biogen Idec, CSL Behring, Kedrion, Novo Nordisk, and Pfizer. The authors report no other conflicts of interest in this work.

\section{References}

1. Collins PW. Management of acquired haemophilia A. JThromb Haemost. 2011;9 Suppl 1:226-235.

2. Cohen AJ, Kessler CM. Acquired inhibitors. Baillieres Clin Haematol. 1996;9(2):331-354.

3. Toschi V, Baudo F. Diagnosis, laboratory aspects and management of acquired hemophilia A. Intern Emerg Med. 2010;5(4):325-333. 
4. Ma AD, Carrizosa D. Acquired factor VIII inhibitors: pathophysiology and treatment. Hematology Am Soc Hematol Educ Program. 2006: 432-437.

5. Collins PW, Hirsch S, Baglin TP, et al; UK Haemophilia Centre Doctors' Organisation. Acquired hemophilia A in the United Kingdom: a 2-year national surveillance study by the United Kingdom Haemophilia Centre Doctors' Organisation. Blood. 2007;109(5):1870-1877.

6. Green D, Lechner K. A survey of 215 non-hemophilic patients with inhibitors to Factor VIII. Thromb Haemost. 1981;45(3):200-203.

7. Delgado J, Jimenez-Yuste V, Hernandez-Navarro F, Villar A. Acquired haemophilia: review and meta-analysis focused on therapy and prognostic factors. Br J Haematol. 2003;121(1):21-35.

8. Knoebl P, Marco P, Baudo F, et al; EACH2 Registry Contributors. Demographic and clinical data in acquired hemophilia A: results from the European Acquired Haemophilia Registry (EACH2). J Thromb Haemost. 2012;10(4):622-631.

9. Borg JY, Guillet B, Le Cam-Duchez V, Goudemand J, Lévesque H; SACHA Study Group. Outcome of acquired haemophilia in France: the prospective SACHA (Surveillance des Auto antiCorps au cours de l'Hémophilie Acquise) registry. Haemophilia. 2013;19(4): 564-570.

10. Webert KE. Acquired hemophilia A. Semin Thromb Hemost. 2012; 38(7):735-741.

11. Coppola A, Favaloro EJ, Tufano A, Di Minno MN, Cerbone AM, Franchini M. Acquired inhibitors of coagulation factors: part I-acquired hemophilia A. Semin Thromb Hemost. 2012;38(5):433-446.

12. Srivastava A, Brewer AK, Mauser-Bunschoten EP, et al; Treatment Guidelines Working Group on Behalf of The World Federation of Hemophilia. Guidelines for the management of hemophilia. Haemophilia. 2013;19(1):e1-e47.

13. Collins P, Baudo F, Huth-Kühne A, et al. Consensus recommendations for the diagnosis and treatment of acquired hemophilia A. BMC Res Notes. 2010;3:161.

14. W Collins P, Chalmers E, Hart D, et al; United Kingdom Haemophilia Centre Doctors' Organization. Diagnosis and management of acquired coagulation inhibitors: a guideline from UKHCDO. Br J Haematol. 2013;162(6):758-773.

15. Huth-Kühne A, Baudo F, Collins $P$, et al. International recommendations on the diagnosis and treatment of patients with acquired hemophilia $\mathrm{A}$ Haematologica. 2009;94(4):566-575.

16. Collins PW. Treatment of acquired hemophilia A. J Thromb Haemost. 2007;5(5):893-900.

17. Shetty S, Bhave M, Ghosh K. Acquired hemophilia a: diagnosis, aetiology, clinical spectrum and treatment options. Autoimmun Rev. 2011; 10(6):311-316.

18. Sborov DW, Rodgers GM. Acquired hemophilia a: a current review of autoantibody disease. Clin Adv Hematol Oncol. 2012;10(1):19-27.

19. Collins PW. Therapeutic challenges in acquired factor VIII deficiency. Hematology Am Soc Hematol Educ Program. 2012;2012:369-374.

20. Franchini M. Postpartum acquired factor VIII inhibitors. Am J Hematol. 2006;81(10):768-773.

21. Zeitler H, Goldmann G, Marquardt N, Ulrich-Merzenich G. Long term outcome of patients with acquired haemophilia - a monocentre interim analysis of 82 patients. Atheroscler Suppl. 2013;14(1):223-228.

22. Turecek PL, Váradi K, Gritsch H, Schwarz HP. FEIBA: mode of action. Haemophilia. 2004;10 Suppl 2:3-9.

23. Hoffman M, Monroe DM 3rd. The action of high-dose factor VIIa (FVIIa) in a cell-based model of hemostasis. Dis Mon. 2003;49(1):14-21.

24. Váradi K, Negrier C, Berntorp E, et al. Monitoring the bioavailability of FEIBA with a thrombin generation assay. J Thromb Haemost. 2003;1(11):2374-2380.

25. Villar A, Aronis S, Morfini M, et al. Pharmacokinetics of activated recombinant coagulation factor VII (NovoSeven) in children vs adults with haemophilia A. Haemophilia. 2004;10(4):352-359.

26. Baudo F, Collins P, Huth-Kühne A, et al; EACH2 registry contributors. Management of bleeding in acquired hemophilia A: results from the European Acquired Haemophilia (EACH2) Registry. Blood. 2012; 120(1):39-46.
27. Astermark J, Donfield SM, DiMichele DM, et al; FENOC Study Group. A randomized comparison of bypassing agents in hemophilia complicated by an inhibitor: the FEIBA NovoSeven Comparative (FENOC) Study. Blood. 2007;109(2):546-551.

28. Holmström M, Tran HT, Holme PA. Combined treatment with APCC $\left(\right.$ FEIBA $\left.^{\circledR}\right)$ and tranexamic acid in patients with haemophilia A with inhibitors and in patients with acquired haemophilia $\mathrm{A}-\mathrm{a}$ two-centre experience. Haemophilia. 2012;18(4):544-549.

29. Aledort LM. Comparative thrombotic event incidence after infusion of recombinant factor VIIa versus factor VIII inhibitor bypass activity. J Thromb Haemost. 2004;2(10):1700-1708.

30. Ehrlich HJ, Henzl MJ, Gomperts ED. Safety of factor VIII inhibitor bypass activity (FEIBA): 10-year compilation of thrombotic adverse events. Haemophilia. 2002;8(2):83-90.

31. O'Connell KA, Wood JJ, Wise RP, Lozier JN, Braun MM. Thromboembolic adverse events after use of recombinant human coagulation factor VIIa. JAMA. 2006;295(3):293-298.

32. Schneiderman J, Rubin E, Nugent DJ, Young G. Sequential therapy with activated prothrombin complex concentrates and recombinant FVIIa in patients with severe haemophilia and inhibitors: update of our previous experience. Haemophilia. 2007;13:244-248.

33. Dehmel H, Werwitzke S, Trummer A, Ganser A, Tiede A. Thrombelastographic monitoring of recombinant factor VIIa in acquired haemophilia. Haemophilia. 2008;14(4):736-742.

34. Hay CR. Porcine factor VIII: past, present and future. Haematologica 2000;85(10 Suppl):21-24; discussion 24-25.

35. Morrison AE, Ludlam CA, Kessler C. Use of porcine factor VIII in the treatment of patients with acquired hemophilia. Blood. 1993;81(6): 1513-1520.

36. Giangrande PL. Porcine factor VIII. Haemophilia. 2012;18(3): 305-309.

37. OBIZUR [prescribing information]. Westlake Village, CA: Baxter Healthcare Corporation; 2014.

38. Muto A, Yoshihashi K, Takeda M, et al. Anti-factor IXa/X bispecific antibody ACE910 prevents spontaneous joint bleeds in a long-term primate model of acquired hemophilia A. Blood. 2014;124(20):3165-3171.

39. Mann KG, Jenny RJ, Krishnaswamy S. Cofactor proteins in the assembly and expression of blood clotting enzyme complexes. Annu Rev Biochem. 1988;57:915-956.

40. Nesheim ME, Taswell JB, Mann KG. The contribution of bovine Factor V and Factor Va to the activity of prothrombinase. J Biol Chem. 1979; 254(21):10952-10962.

41. von Drygalski A, Cramer TJ, Bhat V, Griffin JH, Gale AJ, Mosnier LO. Improved hemostasis in hemophilia mice by means of an engineered factor Va mutant. J Thromb Haemost. 2014;12(3):363-372.

42. Gale AJ, Xu X, Pellequer JL, Getzoff ED, Griffin JH. Interdomain engineered disulfide bond permitting elucidation of mechanisms of inactivation of coagulation factor Va by activated protein C. Protein Sci. 2002;11(9):2091-2101.

43. von Drygalski A, Bhat V, Gale AJ, Griffin JH, Mosnier LO. Synergism of augmented FVa activity and recombinant human FVIIa represent a novel bypassing strategy in hemophilic patients with inhibitors. Haemophilia. 2014;20(Suppl 3):41-42.

44. Hay CR. Acquired haemophilia. Baillieres Clin Haematol. 1998;11(2): 287-303.

45. Lian EC, Larcada AF, Chiu AY. Combination immunosuppressive therapy after factor VIII infusion for acquired factor VIII inhibitor. Ann Intern Med. 1989;110(10):774-778.

46. Baudo F, Caimi T, de Cataldo F. Diagnosis and treatment of acquired haemophilia. Haemophilia. 2010;16(102):102-106.

47. Collins P, Baudo F, Knoebl P, et al; EACH2 registry collaborators. Immunosuppression for acquired hemophilia A: results from the European Acquired Haemophilia Registry (EACH2). Blood. 2012; 120(1):47-55.

48. Green D, Rademaker AW, Briët E. A prospective, randomized trial of prednisone and cyclophosphamide in the treatment of patients with factor VIII autoantibodies. Thromb Haemost. 1993;70(5):753-757.

49. Bitting RL, Bent S, Li Y, Kohlwes J. The prognosis and treatment of acquired hemophilia: a systematic review and meta-analysis. Blood Coagul Fibrinolysis. 2009;20(7):517-523. 
Journal of Blood Medicine

\section{Publish your work in this journal}

The Journal of Blood Medicine is an international, peer-reviewed, open access, online journal publishing laboratory, experimental and clinical aspects of all topics pertaining to blood based medicine including but not limited to: Transfusion Medicine; Blood collection, Donor issues, Transmittable diseases, and Blood banking logistics; Immunohematology; Artificial and alternative

Submit your manuscript here: http://www.dovepress.com/Journal-of-blood-medicine-journal

blood based therapeutics; Hematology; Biotechnology/nanotechnology of blood related medicine; Legal aspects of blood medicine; Historical perspectives. The manuscript management system is completely online and includes a very quick and fair peer-review system. Visit http://www.dovepress.com/ testimonials.php to read real quotes from published authors. 